\title{
Modulation-Mode Assignment in SVD-Aided Downlink Multiuser MIMO-OFDM Systems
}

\author{
Sebastian Aust, Andreas Ahrens, and César Benavente-Peces
}

\begin{abstract}
Multicarrier transmission such as OFDM (orthogonal frequency division multiplexing) is an established technique for radio transmission systems and it can be considered as a promising approach for next generation wireless systems. However, in order to comply with the demand on increasing available data rates in particular in wireless technologies, systems with multiple transmit and receive antennas, also called MIMO (multiple-input multiple-output) systems, have become indispensable for future generations of wireless systems. Due to the strongly increasing demand in high-data rate transmission systems, frequency non-selective MIMO links have reached a state of maturity and frequency selective MIMO links are in the focus of interest. In this field, the combination of MIMO transmission and OFDM can be considered as an essential part of fulfilling the requirements of future generations of wireless systems. However, single-user scenarios have reached a state of maturity. By contrast multiple users' scenarios require substantial further research, where in comparison to $\mathrm{ZF}$ (zero-forcing) multiuser transmission techniques, the individual user's channel characteristics are taken into consideration in this contribution. The performed joint optimization of the number of activated MIMO layers and the number of transmitted bits per subcarrier shows that not necessarily all user-specific MIMO layers per subcarrier have to be activated in order to minimize the overall BER under the constraint of a given fixed data throughput.
\end{abstract}

Keywords-Multiple-input multiple-output system, orthogonal frequency division multiplexing, singular-value decomposition, bit allocation, wireless transmission.

\section{INTRODUCTION}

$\mathbf{M}$ ULTICARRIER transmission represents a suitable method for the digital signal transmission over linear distorting channels [2]-[4]. The available transmission bandwidth is decomposed into a number of narrowband channels in which data streams are transmitted with reduced speed. However, in order to comply with the demand on increasing available data rates in particular in wireless technologies, systems with multiple transmit and receive antennas, also called MIMO (multiple-input multiple-output) systems, have become indispensable and can be considered as an essential part of increasing both the achievable capacity and integrity of future generations of wireless systems [5]-[7]. With the increasing desire for high-data rate communication, frequency non-selective MIMO links have reached a state of maturity. By contrast, frequency selective MIMO links require substantial further research. Spatial-temporal vector coding (STVC) introduced by RALEIGH seems to be an appropriate candidate for broadband transmission channels. Unfortunately, such solutions appear to be highly complex [8], [9]. Therefore, complexity-reduced solutions are of great interest, where multicarrier transmission such as OFDM (orthogonal frequency division multiplexing) combined with multiple transmit and receive antennas seems to be a promising solution to reduce the complexity significantly. The combination of MIMO transmission and OFDM (orthogonal frequency division multiplexing), as investigated in this work, can be considered as an essential part of fulfilling the requirements of future generations of wireless systems. However, single-user scenarios have reached a state of maturity. By contrast multiple users' scenarios in frequency-selective channel conditions require substantial further research [10], [11]. Against this background, in this paper a SVD-assisted multiuser MIMO-ODFM scheme is investigated, where multiuser interferences as well as multi-antenna interferences are perfectly eliminated on each subcarrier. Instead of treating all users' channels jointly as in ZF (zeroforcing) multiuser transmission techniques, the investigated solutions take the individual users' channel characteristics into account [11]. The novel contribution of this paper is that we demonstrate the benefits of amalgamating a suitable choice of activated MIMO layers and number of bits per subcarrier under the constraint of a given fixed data throughput.

The remaining part of this paper is organized as follows: Section II introduces the subcarrier-specific MIMO-OFDM system model, while the proposed optimization objects are discussed in section III. The associated performance results are presented and interpreted in section IV. Finally, section V provides some concluding remarks.

\section{MIMO-OFDM SYSTEM MODEL}

In this section a subcarrier-specific MIMO-OFDM system model for both single-user and multi-user scenarios is developed where the arising subcarrier-specific interferences are perfectly eliminated. The system model considered in this work consists of a single base station (BS) supporting $K$ mobile stations (MSs). The BS is equipped with $n_{\mathrm{T}}$ transmit antennas, while the $k$ th (with $k=1, \ldots, K$ ) MS has $n_{\mathrm{R} k}$ receive antennas, i.e. the total number of receive antennas including all $K \mathrm{MSs}$ is given by $n_{\mathrm{R}}=\sum_{k=1}^{K} n_{\mathrm{R} k}$.

In order to combat the effects of the frequency selective MIMO channel, OFDM is used as transmission technique [12], [2]. Together with a sufficient guard interval length, interferences between the subcarriers can be avoided and only symbols that are transmitted over the different antennas at 
same subcarrier can interfere each other. Thus, the arising multi-antenna and multiuser interferences between the different data streams, transmitted over the same subcarrier, require appropriate subcarrier-specific signal processing strategies.

\section{A. Single-User System}

Considering a single-user MIMO link $(K=1)$ composed of $n_{\mathrm{T}}$ transmit and $n_{\mathrm{R}}$ receive antennas, the obtained $\left(n_{\mathrm{R}}, n_{\mathrm{T}}\right)$ MIMO-OFDM system transmits an $N$-point IFFT ( $N$ subchannels) modulated data signal over every transmit antenna. The subcarrier-specific system is modelled by

$$
\mathbf{u}^{(\kappa)}=\mathbf{H}^{(\kappa)} \cdot \mathbf{c}^{(\kappa)}+\mathbf{n}^{(\kappa)} .
$$

In (1), the $\left(n_{\mathrm{T}} \times 1\right)$ vector $\mathrm{c}^{(\kappa)}$ contains the complex input symbols transmitted over the $\kappa$ th subcarrier on each input. Applying OFDM with a sufficient guard interval length, the $\left(n_{\mathrm{R}} \times n_{\mathrm{T}}\right)$ matrix $\mathbf{H}^{(\kappa)}$ in (1) results in

$$
\mathbf{H}^{(\kappa)}=\left[\begin{array}{ccc}
h_{11}^{(\kappa)} & \cdots & h_{1 n_{\mathrm{T}}}^{(\kappa)} \\
\vdots & \ddots & \vdots \\
h_{n_{\mathrm{R}} 1}^{(\kappa)} & \cdots & h_{n_{\mathrm{R}} n_{\mathrm{T}}}^{(\kappa)}
\end{array}\right],
$$

with the elements $h_{\nu \mu}^{(\kappa)}$ describing the couplings of the data symbols on the subchannel $\kappa$. The elements can be ascertained calculating the FFT of the channel impulse response from transmitter $\mu$ to receiver $\nu$. Finally, $\mathbf{u}^{(\kappa)}$ describes the $\left(n_{\mathrm{R}} \times 1\right)$ received vector and $\mathbf{n}^{(\kappa)}$ is the $\left(n_{\mathrm{R}} \times 1\right)$ vector of the Additive, White Gaussian Noise (AWGN) having a variance of $U_{\mathrm{R}}^{2}$ for both the real and imaginary parts.

The subcarrier-specific interference, introduced by the nondiagonal matrix $\mathbf{H}^{(\kappa)}$, requires appropriate signal processing strategies. A popular technique is based on the singular-value decomposition (SVD) of the matrix $\mathbf{H}^{(\kappa)}$, which can be written as

$$
\mathbf{H}^{(\kappa)}=\mathbf{U}^{(\kappa)} \cdot \mathbf{V}^{(\kappa)} \cdot \mathbf{D}^{(\kappa) \mathrm{H}},
$$

where $\mathbf{U}^{(\kappa)}$ and $\mathbf{D}^{(\kappa)} \mathrm{H}$ are unitary matrices and $\mathbf{V}^{(\kappa)}$ is a real-valued diagonal matrix of the positive square roots of the eigenvalues of the matrix $\mathbf{H}^{(\kappa)} \mathrm{H} \mathbf{H}^{(\kappa)}$ sorted in descending order. The transpose and conjugate transpose (Hermitian) of $\mathbf{D}^{(\kappa)}$ are denoted by $\mathbf{D}^{(\kappa) \mathrm{T}}$ and $\mathbf{D}^{(\kappa)} \mathrm{H}$, respectively. Using $\mathbf{D}^{(\kappa)}$ as preprocessing matrix at the transmitter side and $\mathbf{U}^{(\kappa) H}$ as postprocessing matrix at the receiver side, the overall transmission relationship results in

$$
\begin{aligned}
\mathbf{y}^{(\kappa)} & =\mathbf{U}^{(\kappa) \mathrm{H}}\left(\mathbf{H}^{(\kappa)} \cdot \mathbf{D}^{(\kappa)} \cdot \mathbf{c}^{(\kappa)}+\mathbf{n}^{(\kappa)}\right) \\
& =\mathbf{V}^{(\kappa)} \cdot \mathbf{c}^{(\kappa)}+\mathbf{w}^{(\kappa)} .
\end{aligned}
$$

Thereby, as $\mathbf{D}^{(\kappa)}$ and $\mathbf{U}^{(\kappa) \mathrm{H}}$ are unitary matrices, neither the transmit power nor the noise power is enhanced. The resulting single-user SVD-based MIMO-OFDM system model is represented in Fig. 1. As a consequence of the processing in (4), the subcarrier-specific channel matrix $\mathbf{H}^{(\kappa)}$ is transformed into independent, non-interfering layers having unequal gains. The resulting subcarrier-specific layer-based MIMO-OFDM system model is highlighted in Fig. 2.

The data symbol $c_{1, \ell}^{(\kappa)}$ to be transmitted over the layer $\ell$ (with $\ell=1,2, \ldots, \min \left(n_{\mathrm{T}}, n_{\mathrm{R}}\right)$ ) at the subcarrier $\kappa$ (with $\kappa=$

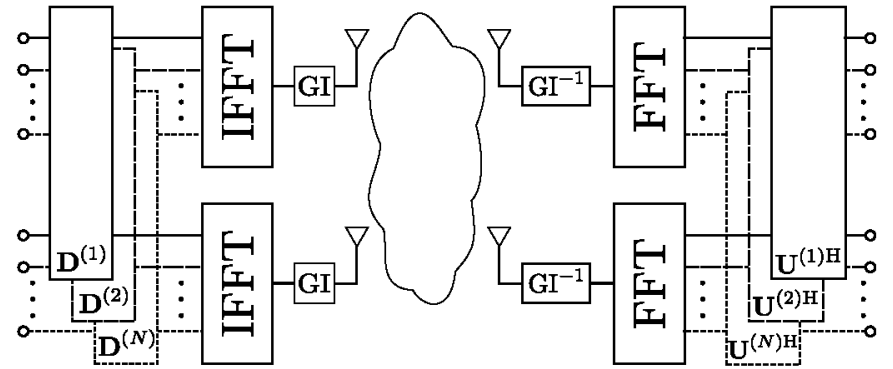

Fig. 1. Resulting single-user SVD-based MIMO-OFDM system model with guard interval (GI).

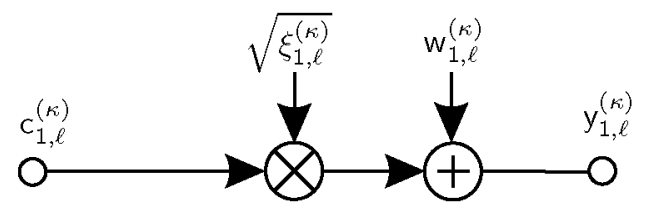

Fig. 2. Resulting subcarrier-specific layer-based single-user MIMO-OFDM system model.

$1,2, \ldots, N)$ is weighted by the layer-specific factor $\sqrt{\xi_{1, \ell}^{(\kappa)}}$, corresponding to the positive square root of the eigenvalue in $\mathbf{V}^{(\kappa)}$, and together with the additive noise term $w_{1, \ell}^{(\kappa)}$ the received data $y_{1, \ell}^{(\kappa)}$ results in

$$
y_{1, \ell}^{(\kappa)}=\sqrt{\xi_{1, \ell}^{(\kappa)}} \cdot c_{1, \ell}^{(\kappa)}+w_{1, \ell}^{(\kappa)}
$$

Therein, the number of easily separable layers per subcarrier $L$ is limited by the minimum numbers of antennas at both sides, the transmitter as well as the receiver side, i.e.,

$$
L \leq \min \left(n_{\mathrm{T}}, n_{\mathrm{R}}\right)
$$

However, it is worth noting that with the aid of powerful nonlinear near Maximum Likelihood (ML) sphere decoders it is possible to separate $n_{\mathrm{R}}>n_{\mathrm{T}}$ number of layers [13].

\section{B. Multi-User System}

Now, the subcarrier-specific single-user MIMO-OFDM system model $(K=1)$ is extended by considering a single base station (BS) supporting $K$ mobile stations (MSs). The $\left(n_{\mathrm{R} k} \times 1\right)$ user specific symbol vector $\mathrm{c}_{k}^{(\kappa)}$ to be transmitted by the BS over the subcarrier $\kappa$ is given by

$$
\mathbf{c}_{k}^{(\kappa)}=\left(c_{k, 1}^{(\kappa)}, c_{k, 2}^{(\kappa)}, \ldots, c_{k, n_{\mathrm{R} k}}^{(\kappa)}\right)^{\mathrm{T}} .
$$

The vector $\mathbf{c}_{k}^{(\kappa)}$ is preprocessed before its transmission by multiplying it with the $\left(n_{\mathrm{T}} \times n_{\mathrm{R} k}\right)$ subcarrier-specific DL preprocessing matrix $\mathbf{R}_{k}^{(\kappa)}$ and results in the $\left(n_{\mathrm{T}} \times 1\right)$ userspecific transmit vector

$$
\mathbf{s}_{k}^{(\kappa)}=\mathbf{R}_{k}^{(\kappa)} \mathbf{c}_{k}^{(\kappa)}
$$

After subcarrier-specific DL transmitter preprocessing, the $n_{\mathrm{T}^{-}}$ component signal $s^{(\kappa)}$ transmitted by the BS specific antennas over the subcarrier $\kappa$ to the $K$ MSs results in

$$
\mathbf{s}^{(\kappa)}=\sum_{k=1}^{K} \mathbf{s}_{k}^{(\kappa)}=\mathbf{R}^{(\kappa)} \mathbf{c}^{(\kappa)}
$$


with the $\left(n_{\mathrm{T}} \times n_{\mathrm{R}}\right)$ preprocessing matrix

$$
\mathbf{R}^{(\kappa)}=\left(\mathbf{R}_{1}^{(\kappa)}, \mathbf{R}_{2}^{(\kappa)}, \ldots, \mathbf{R}_{K}^{(\kappa)}\right)
$$

In (9), the overall $\left(n_{\mathrm{R}} \times 1\right)$ transmitted DL data vector $\mathrm{c}^{(\kappa)}$ combines all $K$ DL transmit vectors $\mathrm{c}_{k}^{(\kappa)}$ (with $k=$ $1,2, \ldots, K)$ and is given by

$$
\mathbf{c}^{(\kappa)}=\left(\mathbf{c}_{1}^{(\kappa) \mathrm{T}}, \mathbf{c}_{2}^{(\kappa) \mathrm{T}} \ldots, \mathbf{c}_{K}^{(\kappa) \mathrm{T}}\right)^{\mathrm{T}}
$$

At the receiver side, the $\left(n_{\mathrm{R} k} \times 1\right)$ vector $\mathbf{u}_{k}^{(\kappa)}$ of the $k$ th MS is given by

$$
\mathbf{u}_{k}^{(\kappa)}=\mathbf{H}_{k}^{(\kappa)} \mathbf{s}^{(\kappa)}+\mathbf{n}_{k}^{(\kappa)}=\mathbf{H}_{k}^{(\kappa)} \mathbf{R}^{(\kappa)} \mathbf{c}^{(\kappa)}+\mathbf{n}_{k}^{(\kappa)} .
$$

and can be expressed by

$$
\mathbf{u}_{k}^{(\kappa)}=\mathbf{H}_{k}^{(\kappa)} \mathbf{R}_{k}^{(\kappa)} \mathbf{c}_{k}^{(\kappa)}+\sum_{i=1, i \neq k}^{K} \mathbf{H}_{k}^{(\kappa)} \mathbf{R}_{i}^{(\kappa)} \mathbf{c}_{i}^{(\kappa)}+\mathbf{n}_{k}^{(\kappa)}
$$

where the MSs received signals at the subcarrier $\kappa$ (with $\kappa=1,2, \ldots, N$ ) experience both multi-user and multi-antenna interferences. In (12), the $\left(n_{\mathrm{R} k} \times n_{\mathrm{T}}\right)$ subcarrier-specific channel matrix $\mathbf{H}_{k}^{(\kappa)}$ connects the $n_{\mathrm{T}} \mathrm{BS}$ specific transmit antennas with the $n_{\mathrm{R} k}$ receive antennas of the $k$ th MS.

The subcarrier-specific interference, which is introduced by the off-diagonal elements of the channel matrix $\mathbf{H}_{k}^{(\kappa)}$, requires appropriate signal processing strategies. A popular technique is based on the SVD of the system matrix $\mathbf{H}_{k}^{(\kappa)}$. Upon carrying out the SVD of $\mathbf{H}_{k}^{(\kappa)}$ with $n_{\mathrm{T}} \geq n_{\mathrm{R}}$ and assuming that the rank of the matrix $\mathbf{H}_{k}^{(\kappa)}$ equals $n_{\mathrm{R} k}$, i. e., $\operatorname{rank}\left(\mathbf{H}_{k}^{(\kappa)}\right)=n_{\mathrm{R} k}$, we get

$$
\mathbf{H}_{k}^{(\kappa)}=\mathbf{U}_{k}^{(\kappa)} \cdot \mathbf{V}_{k}^{(\kappa)} \cdot \mathbf{D}_{k}^{(\kappa) \mathrm{H}}
$$

with the $\left(n_{\mathrm{R} k} \times n_{\mathrm{R} k}\right)$ unitary matrix $\mathbf{U}_{k}^{(\kappa)}$ and the $\left(n_{\mathrm{T}} \times n_{\mathrm{T}}\right)$ unitary matrix $\mathbf{D}_{k}^{(\kappa) \mathrm{H}}$, respectively. The $\left(n_{\mathrm{R} k} \times n_{\mathrm{T}}\right)$ diagonal matrix $\mathbf{V}_{k}^{(\kappa)}$ can be decomposed into a $\left(n_{\mathrm{R} k} \times n_{\mathrm{R} k}\right)$ matrix $\mathbf{V}_{k \mathrm{u}}^{(\kappa)}$ containing the non-zero square roots of the eigenvalues of $\mathbf{H}_{k}^{(\kappa) \mathrm{H}} \mathbf{H}_{k}^{(\kappa)}$, i. e.,

$$
\mathbf{V}_{k \mathrm{u}}^{(\kappa)}=\left[\begin{array}{cccc}
\sqrt{\xi_{k, 1}^{(\kappa)}} & 0 & \cdots & 0 \\
0 & \sqrt{\xi_{k, 2}^{(\kappa)}} & \ddots & \vdots \\
\vdots & \ddots & \ddots & \vdots \\
0 & 0 & \cdots & \sqrt{\xi_{k, n_{\mathrm{R} k}}^{(\kappa)}}
\end{array}\right]
$$

and a $\left(n_{\mathrm{R} k} \times\left(n_{\mathrm{T}}-n_{\mathrm{R} k}\right)\right)$ zero-matrix $\mathbf{V}_{k \mathrm{n}}^{(\kappa)}$ according to

$$
\mathbf{V}_{k}^{(\kappa)}=\left(\mathbf{V}_{k \mathrm{u}}^{(\kappa)} \mathbf{V}_{k \mathrm{n}}^{(\kappa)}\right)=\left(\mathbf{V}_{k \mathrm{u}}^{(\kappa)} \mathbf{0}\right)
$$

Additionally, the $\left(n_{\mathrm{T}} \times n_{\mathrm{T}}\right)$ unitary matrix $\mathbf{D}_{k}^{(\kappa)}$ can be decomposed into a $\left(n_{\mathrm{T}} \times n_{\mathrm{R} k}\right)$ matrix $\mathbf{D}_{k \mathrm{u}}^{(\kappa)}$ constituted by the eigenvectors corresponding to the non-zero eigenvalues of $\mathbf{H}_{k}^{(\kappa) \mathrm{H}} \mathbf{H}_{k}^{(\kappa)}$ and a $\left(n_{\mathrm{T}} \times\left(n_{\mathrm{T}}-n_{\mathrm{R} k}\right)\right)$ matrix $\mathbf{D}_{k \mathrm{n}}^{(\kappa)}$ constituted by the eigenvectors corresponding to the zero eigenvalues of $\mathbf{H}_{k}^{(\kappa) \mathrm{H}} \mathbf{H}_{k}^{(\kappa)}$. The decomposition of the matrix $\mathbf{D}_{k}^{(\kappa) \mathrm{H}}$ results in

$$
\mathbf{D}_{k}^{(\kappa) \mathrm{H}}=\left(\begin{array}{c}
\mathbf{D}_{k \mathrm{u}}^{(\kappa) \mathrm{H}} \\
\mathbf{D}_{k \mathrm{n}}^{(\kappa) \mathrm{H}}
\end{array}\right)
$$

Finally, the subcarrier-specific downlink received signal $\mathbf{u}_{k}^{(\kappa)}$ of the $k$ th MS may be expressed as

$$
\mathbf{u}_{k}^{(\kappa)}=\mathbf{U}_{k}^{(\kappa)} \mathbf{V}_{k \mathrm{u}}^{(\kappa)} \mathbf{D}_{k \mathrm{u}}^{(\kappa) \mathrm{H}} \mathbf{R}^{(\kappa)} \mathbf{c}^{(\kappa)}+\mathbf{n}_{k}^{(\kappa)}
$$

with the vector $\mathbf{n}_{k}^{(\kappa)}$ of the Additive, White Gaussian Noise (AWGN). Taking all MSs received DL signals $\mathbf{u}_{k}^{(\kappa)}$ into account, the $\left(n_{\mathrm{R}} \times 1\right)$ receive vector results in

$$
\mathbf{u}^{(\kappa)}=\left(\mathbf{u}_{1}^{(\kappa) \mathrm{T}}, \mathbf{u}_{2}^{(\kappa) \mathrm{T}}, \ldots, \mathbf{u}_{K}^{(\kappa) \mathrm{T}}\right)^{\mathrm{T}}
$$

The overall DL signal vector $\mathbf{u}^{(\kappa)}$ including the received signals of all $K$ MSs can be expressed by

$$
\mathbf{u}^{(\kappa)}=\mathbf{U}^{(\kappa)} \mathbf{V}_{\mathrm{u}}^{(\kappa)} \mathbf{D}_{\mathrm{u}}^{(\kappa) \mathrm{H}} \mathbf{R}^{(\kappa)} \mathbf{c}^{(\kappa)}+\mathbf{n}^{(\kappa)},
$$

with the overall $\left(n_{\mathrm{R}} \times 1\right)$ noise vector

$$
\mathbf{n}^{(\kappa)}=\left(\mathbf{n}_{1}^{(\kappa) \mathrm{T}}, \mathbf{n}_{2}^{(\kappa) \mathrm{T}}, \ldots, \mathbf{n}_{K}^{(\kappa) \mathrm{T}}\right)^{\mathrm{T}}
$$

the $\left(n_{\mathrm{R}} \times n_{\mathrm{R}}\right)$ block diagonal matrix $\mathbf{U}^{(\kappa)}$

$$
\mathbf{U}^{(\kappa)}=\left[\begin{array}{cccc}
\mathbf{U}_{1}^{(\kappa)} & \mathbf{0} & \cdots & \mathbf{0} \\
\mathbf{0} & \mathbf{U}_{2}^{(\kappa)} & \ddots & \vdots \\
\vdots & \ddots & \ddots & \vdots \\
0 & 0 & \cdots & \mathbf{U}_{K}^{(\kappa)}
\end{array}\right]
$$

the $\left(n_{\mathrm{R}} \times n_{\mathrm{R}}\right)$ block diagonal matrix $\mathbf{V}_{\mathrm{u}}^{(\kappa)}$

$$
\mathbf{V}_{\mathrm{u}}^{(\kappa)}=\left[\begin{array}{cccc}
\mathbf{V}_{1 \mathrm{u}}^{(\kappa)} & \mathbf{0} & \cdots & \mathbf{0} \\
\mathbf{0} & \mathbf{V}_{2 \mathrm{u}}^{(\kappa)} & \ddots & \vdots \\
\vdots & \ddots & \ddots & \vdots \\
\mathbf{0} & \mathbf{0} & \ldots & \mathbf{V}_{K \mathrm{u}}^{(\kappa)}
\end{array}\right]
$$

and the $\left(n_{\mathrm{T}} \times n_{\mathrm{R}}\right)$ matrix $\mathbf{D}_{\mathrm{u}}^{(\kappa)}$ which is given by

$$
\mathbf{D}_{\mathrm{u}}^{(\kappa)}=\left(\mathbf{D}_{1 \mathrm{u}}^{(\kappa)}, \mathbf{D}_{2 \mathrm{u}}^{(\kappa)}, \ldots, \mathbf{D}_{K \mathrm{u}}^{(\kappa)}\right) .
$$

In order to suppress the DL multiuser interferences (MUI) at the subcarrier $\kappa$ perfectly, the DL preprocessing matrix $\mathbf{R}^{(\kappa)}$ has to be designed to satisfy the following condition

$$
\mathbf{D}_{\mathrm{u}}^{(\kappa) \mathrm{H}} \mathbf{R}^{(\kappa)}=\mathbf{P}^{(\kappa)},
$$

with the real-valued $\left(n_{\mathrm{R}} \times n_{\mathrm{R}}\right)$ diagonal matrix $\mathbf{P}^{(\kappa)}$ taking the transmit-power constraint into account. In order to satisfy (25), $\mathbf{R}^{(\kappa)}$ can be defined as follows

$$
\mathbf{R}^{(\kappa)}=\mathbf{D}_{\mathrm{u}}^{(\kappa)}\left(\mathbf{D}_{\mathrm{u}}^{(\kappa) \mathrm{H}} \mathbf{D}_{\mathrm{u}}^{(\kappa)}\right)^{-1} \mathbf{P}^{(\kappa)}
$$

Taking the ZF design criterion for the DL preprocessing matrix into account, the matrix $\mathbf{P}^{(\kappa)}$ simplifies to an $\left(n_{\mathrm{R}} \times n_{\mathrm{R}}\right)$ diagonal matrix, i. e. $\mathbf{P}^{(\kappa)}=\sqrt{\beta^{(\kappa)}} \mathbf{I}_{n_{\mathrm{R}} \times n_{\mathrm{R}}}$, with the parameter $\sqrt{\beta^{(\kappa)}}$ describing the transmit-power constraint. When taking the DL preprocessing matrix, defined in (26), into account, the overall subcarrier-specific received vector of all $K \mathrm{MSs}$, defined in (20), can be simplified to

$$
\mathbf{u}^{(\kappa)}=\mathbf{U}^{(\kappa)} \mathbf{V}_{\mathrm{u}}^{(\kappa)} \mathbf{P}^{(\kappa)} \mathbf{c}^{(\kappa)}+\mathbf{n}^{(\kappa)}
$$


Therein, the $\left(n_{\mathrm{R}} \times n_{\mathrm{R}}\right)$ block diagonal matrix $\mathbf{P}^{(\kappa)}$ is given by

$$
\mathbf{P}^{(\kappa)}=\left[\begin{array}{cccc}
\mathbf{P}_{1}^{(\kappa)} & \mathbf{0} & \cdots & \mathbf{0} \\
0 & \mathbf{P}_{2}^{(\kappa)} & \ddots & \vdots \\
\vdots & \ddots & \ddots & \vdots \\
0 & 0 & \cdots & \mathbf{P}_{K}^{(\kappa)}
\end{array}\right]
$$

In (27), the user-specific $\left(n_{\mathrm{R} k} \times 1\right)$ vector $\mathbf{u}_{k}^{(\kappa)}$ can be expressed as

$$
\mathbf{u}_{k}^{(\kappa)}=\mathbf{U}_{k}^{(\kappa)} \mathbf{V}_{k \mathrm{u}}^{(\kappa)} \mathbf{P}_{k}^{(\kappa)} \mathbf{c}_{k}^{(\kappa)}+\mathbf{n}_{k}^{(\kappa)},
$$

with the user-specific $\left(n_{\mathrm{R} k} \times n_{\mathrm{R} k}\right)$ power allocation matrix

$$
\mathbf{P}_{k}^{(\kappa)}=\left[\begin{array}{cccc}
\sqrt{p_{k, 1}^{(\kappa)}} & 0 & \cdots & 0 \\
0 & \sqrt{p_{k, 2}^{(\kappa)}} & \ddots & \vdots \\
\vdots & \ddots & \ddots & \vdots \\
0 & 0 & \cdots & \sqrt{p_{k, n_{\mathrm{R} k}}^{(\kappa)}}
\end{array}\right]
$$

As long as the transmit power is uniformly distributed over the number of activated MIMO layers per subcarrier, the matrix $\mathbf{P}_{k}^{(\kappa)}$ simplifies to

$$
\mathbf{P}_{k}^{(\kappa)}=\sqrt{\beta^{(\kappa)}} \mathbf{I}_{n_{\mathrm{R} k} \times n_{\mathrm{R} k}} .
$$

After postprocessing of the received signal vectors $\mathbf{u}_{k}^{(\kappa)}$ with the corresponding unitary matrix $\mathbf{U}_{k}^{(\kappa) H}$, the user-specific decision variables at the subcarrier $\kappa$ result with $\mathbf{U}_{k}^{(\kappa) \mathrm{H}} \mathbf{n}_{k}^{(\kappa)}=$ $\mathbf{w}_{k}^{(\kappa)}$ in

$$
\mathbf{y}_{k}^{(\kappa)}=\mathbf{U}_{k}^{(\kappa) \mathrm{H}} \mathbf{u}_{k}^{(\kappa)}=\mathbf{V}_{k \mathrm{u}}^{(\kappa)} \mathbf{P}_{k}^{(\kappa)} \mathbf{c}_{k}^{(\kappa)}+\mathbf{w}_{k}^{(\kappa)},
$$

or alternatively for the whole system with $\mathbf{U}^{(\kappa) \mathrm{H}} \mathbf{n}^{(\kappa)}=\mathbf{w}^{(\kappa)}$ in

$$
\mathbf{y}^{(\kappa)}=\mathbf{U}^{(\kappa) \mathrm{H}} \mathbf{u}^{(\kappa)}=\mathbf{V}_{\mathrm{u}}^{(\kappa)} \mathbf{P}^{(\kappa)} \mathbf{c}^{(\kappa)}+\mathbf{w}^{(\kappa)},
$$

where subcarrier-specific interferences between the different antenna data streams as well as MUI imposed by the other users are avoided. The resulting multiuser SVD-based MIMOOFDM system model is represented in Fig. 3, whereas the resulting layer-specific system model is depicted in Fig. 4

\section{OPTIMIZATION OBJECTIVES}

In general, the user-specific quality of data transmission can be informally assessed by using the signal-to-noise ratio (SNR) at the detector's input defined by the half vertical eye opening and the noise power per quadrature component according to

$$
\varrho=\frac{(\text { Half vertical eye opening })^{2}}{\text { Noise Power }}=\frac{\left(U_{\mathrm{A}}\right)^{2}}{\left(U_{\mathrm{R}}\right)^{2}},
$$

which is often used as a quality parameter [10], [14]. relationship between the signal-to-noise ratio $\varrho=U_{\mathrm{A}}^{2} / U_{\mathrm{R}}^{2}$ and the bit-error probability evaluated for AWGN channels and $M$-ary Quadrature Amplitude Modulation (QAM) is given by [15]

$$
P_{\mathrm{BER}}=\frac{2}{\log _{2}(M)}\left(1-\frac{1}{\sqrt{M}}\right) \operatorname{erfc}\left(\sqrt{\frac{\varrho}{2}}\right) .
$$

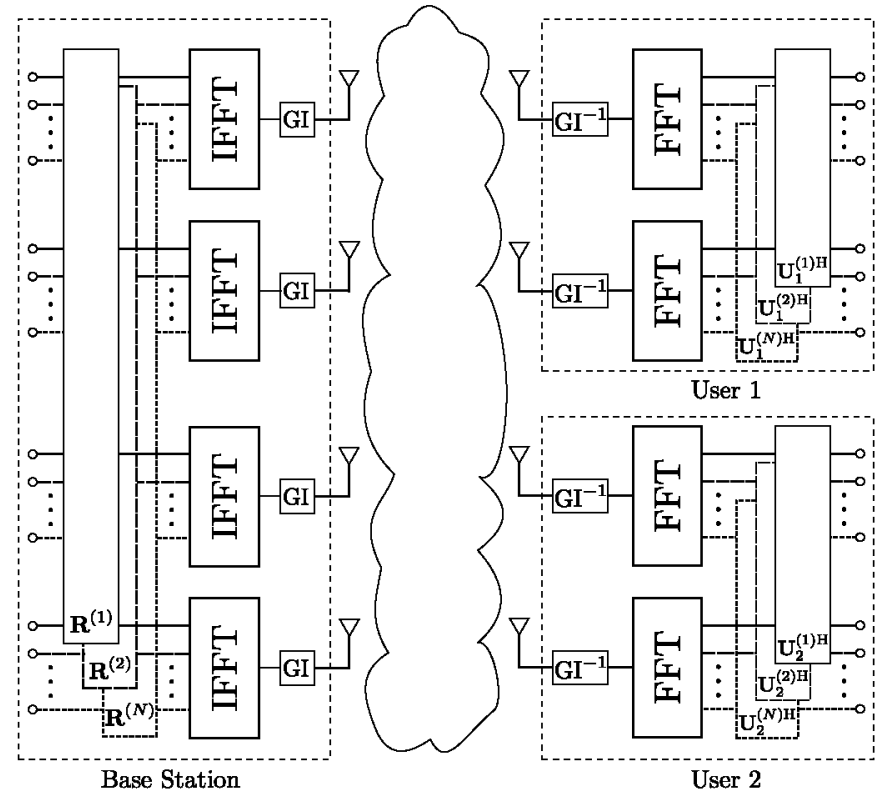

Fig. 3. Resulting multiuser SVD-based MIMO-OFDM system model with guard interval (GI).

When applying the proposed system structure for the $k$ th user, depicted in Fig. 4, the applied signal processing leads to different eye openings per activated MIMO layer $\ell$ (with $\ell=1,2, \ldots, L$ and $L \leq n_{\mathrm{R} k}$ describing the number of activated user-specific MIMO layers) and per subcarrier $\kappa$ (with $\kappa=1,2, \ldots, N$ ) according to

$$
U_{\mathrm{A} k \ell}^{(\kappa)}=\sqrt{p_{k, \ell}^{(\kappa)}} \cdot \sqrt{\xi_{k, \ell}^{(\kappa)}} \cdot U_{\mathrm{s} k \ell}^{(\kappa)},
$$

where $U_{\mathrm{s} k \ell}^{(\kappa)}$ denotes the $k$ th user and $\kappa$ th subcarrier specific half-level transmit amplitude assuming $M_{\ell}$-ary QAM, $\sqrt{\xi_{k, \ell}^{(\kappa)}}$ represents the corresponding subcarrier-specific positive square roots of the eigenvalues of the matrix $\mathbf{H}_{k}^{(\kappa) \mathrm{H}} \mathbf{H}_{k}^{(\kappa)}$ and $\sqrt{p_{k, \ell}^{(\kappa)}}$ represents the corresponding power allocation weighting parameters (Fig. 4). Together with the noise power per quadrature component, introduced by the additive, white Gaussian noise (AWGN) vector $\mathbf{U}_{k}^{(\kappa) \mathrm{H}} \mathbf{n}_{k}^{(\kappa)}=\mathbf{w}_{k}^{(\kappa)}$ in (32), the $k$ th user-specific SNR per MIMO layer $\ell$ and subcarrier $\kappa$ becomes

$$
\varrho_{k \ell}^{(\kappa)}=\frac{\left(U_{\mathrm{A} k \ell}^{(\kappa)}\right)^{2}}{U_{\mathrm{R}}^{2}} .
$$

Realizing a parallel transmission over $L$ MIMO layers and taking all $N$ subcarriers into account, the overall mean user-

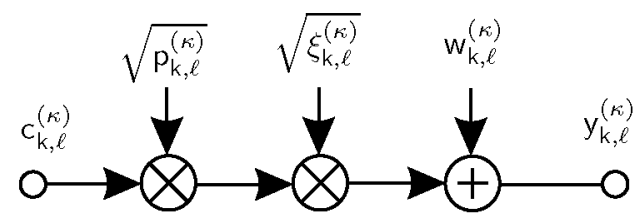

Fig. 4. Resulting $k$ th user system model per MIMO layer $\ell$ (with $\ell=$ $1,2, \ldots, n_{\mathrm{R} k}$ ) on subcarrier $\kappa$ (with $\kappa=1,2, \ldots, N$ ). 
specific transmit power becomes $P_{\mathrm{s} k}=\sum_{\kappa=1}^{N} \sum_{\ell=1}^{L} P_{\mathrm{s} k \ell}^{(\kappa)}$. Considering QAM constellations, the average user-specific transmit power $P_{\mathrm{s} k \ell}^{(\kappa)}$ per MIMO layer $\ell$ and subcarrier $\kappa$ may be expressed as [15]

$$
P_{\mathrm{s} k \ell}^{(\kappa)}=\frac{2}{3}\left(U_{\mathrm{s} k \ell}^{(\kappa)}\right)^{2}\left(M_{k \ell}^{(\kappa)}-1\right) .
$$

Combining (37) and (38) together with (36), the layer-specific SNR at the subcarrier $\kappa$ results in

$$
\varrho_{k \ell}^{(\kappa)}=p_{k, \ell}^{(\kappa)} \xi_{k, \ell}^{(\kappa)} \frac{3}{2\left(M_{k \ell}^{(\kappa)}-1\right)} \frac{P_{\mathrm{s} k \ell}^{(\kappa)}}{U_{\mathrm{R}}^{2}} .
$$

Assuming that the user-specific transmit power is uniformly distributed over the number of activated MIMO layers and subcarriers, the quality on each subcarrier is affected by both, the choice of the QAM-constellation sizes per layer and the layerspecific weighting factors. In order to transmit at a fixed data rate while maintaining the best possible integrity, i.e., bit-error rate, an appropriate number of user-specific MIMO layers has to be used, which depends on the specific transmission mode, as detailed in Table III for the exemplarily investigated twouser system $\left(n_{\mathrm{R} k}=4\right.$ (with $\left.k=1,2\right), K=2, n_{\mathrm{R}}=n_{\mathrm{T}}=8$ ). An optimized adaptive scheme would now use the particular

TABLE I

INVESTIGATED USER-SPECIFIC TRANSMISSION MOdES PER SUBCARRIER

\begin{tabular}{ccccc}
\hline \hline throughput & layer 1 & layer 2 & layer 3 & layer 4 \\
\hline $8 \mathrm{bit} / \mathrm{s} / \mathrm{Hz}$ & 256 & 0 & 0 & 0 \\
$8 \mathrm{bit} / \mathrm{s} / \mathrm{Hz}$ & 64 & 4 & 0 & 0 \\
$\mathbf{8} \mathrm{bit} / \mathrm{s} / \mathrm{Hz}$ & $\mathbf{1 6}$ & $\mathbf{1 6}$ & 0 & 0 \\
$\mathbf{8} \mathrm{bit} / \mathrm{s} / \mathrm{Hz}$ & $\mathbf{1 6}$ & $\mathbf{4}$ & $\mathbf{4}$ & 0 \\
$8 \mathrm{bit} / \mathrm{s} / \mathrm{Hz}$ & 4 & 4 & 4 & 4 \\
\hline \hline
\end{tabular}

transmission modes on each subcarrier, e. g., by using bit auction procedures, that results in the lowest BER for each MIMO-OFDM data vector [16]. However, this would lead to a high signaling overhead. Therefore, in order to avoid signalling overhead, fixed transmission modes are used in this contribution regardless of the channel quality.

\section{RESULTS}

In this contribution the efficiency of fixed user-specific transmission modes on each subcarrier is studied regardless of the channel quality. Assuming predefined transmission modes, a fixed data rate can be guaranteed. In order to obtain numerical results for the analyzed subcarrier-specific signal processing strategies, a two-path channel model is investigated under time-variant conditions between any given transmit and receive antenna combination. The exemplary impulse response between the $\mu$ th transmit and $\nu$ th receive antenna as a function of the multicarrier symbol duration $T_{\mathrm{s}}$ is given by

$$
g_{\mathrm{k}}^{(\nu \mu)}(t, \tau)=g_{\mathrm{k}, 0}(t) \cdot \delta(\tau)+g_{\mathrm{k}, 1}(t) \cdot \delta\left(\tau-3 / 2 T_{\mathrm{s}}\right),
$$

whereby the time-variant behaviour is described by $g_{\mathrm{k}, \mathrm{o}}(t)$ and $g_{\mathrm{k}, 1}(t)$, respectively. It is assumed that the path amplitudes have the same averaged power and undergo a Rayleigh distribution [17]. Furthermore, a block fading channel model is applied, i.e., the channel is assumed to be time invariant

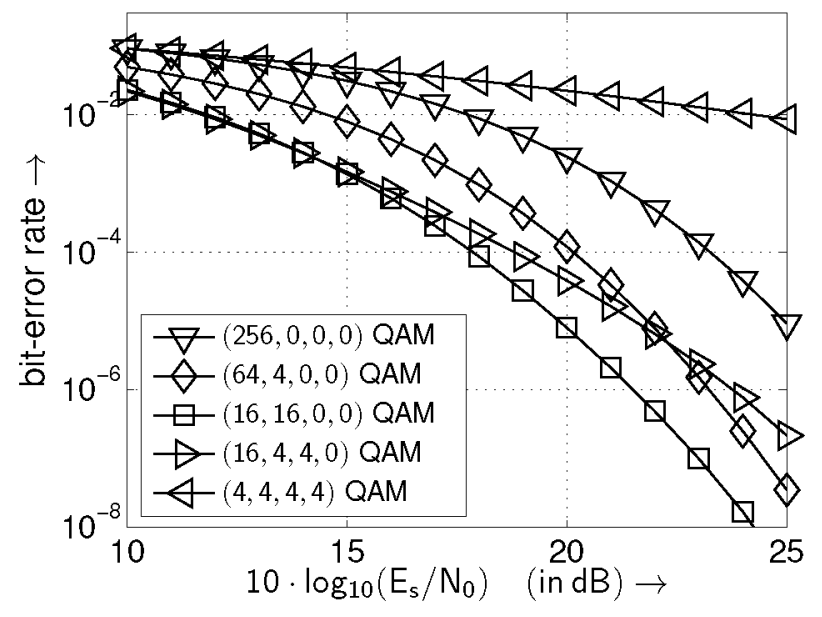

Fig. 5. BER when using the transmission modes introduced in Tab. III and transmitting $8 \mathrm{bit} / \mathrm{s} / \mathrm{Hz}$ per subcarrier.

for the duration of one MIMO-OFDM data vector including a guard interval length of $T_{\mathrm{g}}=T_{\mathrm{s}} / 2$. The number of subcarrier of the investigated OFDM system was exemplarily chosen to be $N=4$.

\section{A. Single-User System}

Considering a single-user MIMO-OFDM link $(K=1)$ composed of $n_{\mathrm{T}}=4$ transmit and $n_{\mathrm{R}}=4$ receive antennas, the corresponding calculated BER curves are depicted in Fig. 5 for the different subcarrier-specific QAM constellation sizes and MIMO configurations of Table III, when transmitting at a fixed bandwidth efficiency of $8 \mathrm{bit} / \mathrm{s} / \mathrm{Hz}$ per subcarrier. Assuming a uniform distribution of the transmit power over the number of activated MIMO layers per subcarrier, it still turns out that not all subcarrier-specific MIMO layers have to be activated in order to achieve the best system performance.

However, the lowest BERs can only be achieved by using bit auction procedures leading to a high signalling overhead [16]. Analyzing the probability of choosing subcarrier-specific transmission modes by using optimal bitloading, as depicted in Table II, it turns out that only an appropriate number of subcarrier-specific MIMO layers has to be activated to reach the best results, e.g., the $(16,4,4,0)$ QAM configuration. Therefore, the results, obtained by using bit auction procedures, justify the choice of fixed subcarrier-specific transmission modes regardless of the channel quality as investigated in this contribution. Further improvements can be achieved by taking power allocation into account [18]-[20].

TABLE II

Probability of Choosing SubCaRRier-S Pecific Transmission Modes at a Fixed Data Rate by Using Optimal Bitloading $\left(10 \cdot \lg \left(E_{s} / N_{0}\right)=10 \mathrm{~dB}\right.$ AND $\left.\kappa=3\right)$

\begin{tabular}{ccccc}
\hline \hline mode & $(16,4,4,0)$ & $(16,16,0,0)$ & $(64,4,0,0)$ & $(4,4,4,4)$ \\
\hline pdf & 0.722 & 0.261 & 0.016 & 0.000 \\
\hline \hline
\end{tabular}




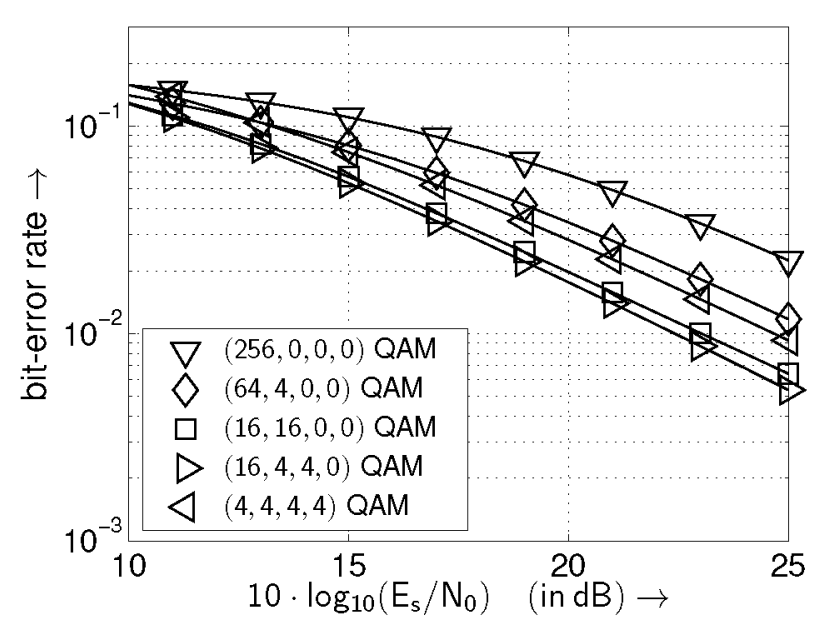

Fig. 6. User-specific BERs when using the transmission modes introduced in Table III and transmitting $8 \mathrm{bit} / \mathrm{s} / \mathrm{Hz}$ per subcarrier.

\section{B. Multi-User System}

The parameters of the analyzed two-users MIMO system are chosen as follows: $P_{\mathrm{s} k}=1 \mathrm{~V}^{2}, n_{\mathrm{R} k}=4$ (with $k=1,2$ ), $K=2, n_{\mathrm{R}}=n_{\mathrm{T}}=8$. In this contribution a power with the dimension (voltage) ${ }^{2}$ (in $\mathrm{V}^{2}$ ) is used. At a real constant resistor this value is proportional to the physical power (in W).

The obtained user-specific BER curves are depicted in Fig. 6 for the different subcarrier-specific QAM constellation sizes and MIMO configurations of Table III and confirm the obtained results within the single-user system $(K=1)$. Based on the higher total subcarrier-specific throughput within the given bandwidth compared to the single-user system, the gap between the different transmission modes becomes smaller. Assuming a uniform distribution of the transmit power over the number of activated MIMO layers, it still turns out that not all MIMO layers per subcarrier have to be activated in order to achieve the best BERs.

This can still be confirmed by analyzing the probability of choosing user-specific transmission modes within the multiuser DL MIMO-OFDM system by using optimal bitloading [16], as depicted in Table III.

TABLE III

Probability of Choosing User-SPECIFIC Transmission Modes $(K=2)$ Per Subcarrier at a Fixed Data Rate by Using Optimal BitLOADING $\left(10 \cdot \log _{10}\left(E_{\mathrm{s}} / N_{0}\right)=10 \mathrm{~dB}\right.$ AND $\left.\kappa=3\right)$

\begin{tabular}{ccccc}
\hline \hline mode & $(16,4,4,0)$ & $(16,16,0,0)$ & $(64,4,0,0)$ & $(4,4,4,4)$ \\
\hline pdf & 0.950 & 0.010 & 0.000 & 0.039 \\
\hline \hline
\end{tabular}

\section{CONCLUSION}

In this paper, the DL performance of a multiuser MIMOOFDM system is investigated theoretically and by software simulation. Frequency selective MIMO channels are considered and conditions to eliminate multiuser and multi-antenna interferences on each subcarrier are established using the SVD of the individual user subcarrier-specific channel matrix. Furthermore, bit allocation in multiuser MIMO-OFDM systems was investigated for constant data throughput per subcarrier. Here, it turned out that the choice of the number of bits per symbol as well as the number of activated MIMO layers per subcarrier substantially affects the performance of a MIMOOFDM system, suggesting that not all user-specific MIMO layers per subcarrier have to be activated in order to achieve the best BERs.

\section{REFERENCES}

[1] S. Aust, A. Ahrens, and C. Benavente-Peces, "Modulation-Mode Assignment in SVD-aided Downlink Multiuser MIMO-OFDM Systems," in Signal Processing Symposium (SPS), Jachranka (Poland), 08- 10 June 2011.

[2] J. A. C. Bingham, ADSL, VDSL, and Multicarrier Modulation. New York: Wiley, 2000.

[3] R. van Nee and R. Prasad, OFDM for Wireless Multimedia Communications. Boston and London: Artech House, 2000.

[4] T. J. Hwang, H. S. Hwang, and H. K. Balik, "Adaptive OFDM with Channel Predictor over Frequency-Selective and Rapid Fading Channel," in Personal, Indoor and Mobile Radio Communications (PIMRC), Bejing (China), 7-10 September 2003, pp. 859-863.

[5] L. Zheng and D. N. T. Tse, "Diversity and Multiplexing: A Fundamental Tradeoff in Multiple-Antenna Channels," IEEE Transactions on Information Theory, vol. 49, no. 5, pp. 1073-1096, May 2003.

[6] V. Kühn, Wireless Communications over MIMO Channels - Applications to CDMA and Multiple Antenna Systems. Chichester: Wiley, 2006.

[7] Z. Zhou, B. Vucetic, M. Dohler, and Y. Li, "MIMO Systems with Adaptive Modulation." IEEE Transactions on Vehicular Technology, vol. 54, no. 5, pp. 1073-1096, September 2005.

[8] G. G. Raleigh and J. M. Cioffi, "Spatio-Temporal Coding for Wireless Communication," IEEE Transactions on Communications, vol. 46, no. 3, pp. 357-366, March 1998.

[9] G. G. Raleigh and V. K. Jones, "Multivariate Modulation and Coding for Wireless Communication," IEEE Journal on Selected Areas in Communications, vol. 17, no. 5, pp. 851-866, May 1999.

[10] A. Ahrens and C. Benavente-Peces, "Modulation-Mode and Power Assignment for SVD-assisted and Iteratively Detected Downlink Multiuser MIMO Systems," in International Conference on Wireless Information Networks and Systems (WINSYS), Athens (Greece), 26-28 July 2010, pp. 107-114.

[11] W. Liu, L. L. Yang, and L. Hanzo, "SVD Assisted Joint Transmitter and Receiver Design for the Downlink of MIMO Systems," in IEEE 68th Vehicular Technology Conference (VTC), Calgary, 2008, pp. 1-5.

[12] A. R. S. Bahai and B. R. Saltzberg, Multi-Carrier Digital Communications - Theory and Applications of OFDM. New York, Boston, Dordrecht, London, Moskau: Kluwer Academic/Plenum Publishers, 1999.

[13] L. Hanzo and T. Keller, OFDM and MC-CDMA. New York: Wiley, 2006.

[14] A. Ahrens and C. Lange, "Modulation-Mode and Power Assignment in SVD-equalized MIMO Systems," Facta Universitatis (Series Electronics and Energetics), vol. 21, no. 2, pp. 167-181, August 2008.

[15] J. G. Proakis, Digital Communications. Boston: McGraw-Hill, 2000.

[16] C. Y. Wong, R. S. Cheng, K. B. Letaief, and R. D. Murch, "Multiuser OFDM with Adaptive Subcarrier, Bit, and Power Allocation," IEEE Journal on Selected Areas in Communications, vol. 17, no. 10, pp. 17471758, October 1999.

[17] M. Pätzold, Mobile Fading Channels. Chichester: Wiley, 2002.

[18] B. S. Krongold, K. Ramchandran, and D. L. Jones, "Computationally Efficient Optimal Power Allocation Algorithms for Multicarrier Communications Systems," IEEE Transactions on Communications, vol. 48 no. 1 , pp. 23-27, 2000.

[19] J. Jang and K. B. Lee, "Transmit Power Adaptation for Multiuser OFDM Systems," IEEE Journal on Selected Areas in Communications, vol. 21, no. 2, pp. 171-178, 2003.

[20] C. S. Park and K. B. Lee, "Transmit Power Allocation for BER Performance Improvement in Multicarrier Systems," IEEE Transactions on Communications, vol. 52, no. 10, pp. 1658-1663, 2004. 